\title{
THE RELATIONSHIP OF ASSISTED REPRODUCTIVE TECHNOLOGY WITH COVID-19 INFECTION IN WOMEN
}

\author{
Yeliz Kaya ${ }^{1}$, Yavuz Tokgöz ${ }^{2}$, Coskun Kaya ${ }^{3}$, Tugba Tahta ${ }^{2}$, and Başar Tekin ${ }^{2}$ \\ ${ }^{1}$, Eskişehir Osmangazi University Faculty of Health Sciences, \\ ${ }^{2}$ Affiliation not available \\ ${ }^{3}$ Eskisehir City Hospital, Department of Urology
}

September 11, 2020

\begin{abstract}
Backgrounds: To investigate the association between assisted reproductive technology (ART) treatments and the Coronavirus Disease 2019 (COVID-19). Methods: The data of the 17 infertility patients who were treated with ART between 01 and 11 March 2020 at a Reproductive Endocrinology and Infertility Unit of a tertiary center were collected retrospectively. The patients were then asked if they were positive for the COVID-19 or had symptoms of the illness. Results: Ovarian stimulation, oocyte pick-up, and intracytoplasmic sperm injection were used for all of the patients. Nine (52.9\%) patients' embryos were transferred and pregnancy was detected in 4 of the 9 patients after the assisted reproductive treatments. At the time of the interview, none of the infertile women had any symptoms of the COVID-19 or a confirmed diagnosis from a positive test result for the COVID-19 nucleic acids by real-time fluorescence reverse transcription-polymerase chain reaction. Conclusion: When turning to normal practice life, all patients should be evaluated for COVID-19 during pre, per and post-treatment time in order to stop the ART and protect against the cases from the possible side effects of assisted reproductive treatments, especially the possible, but undefined, predisposition factor to COVID-19.
\end{abstract}

\section{Introduction}

The World Health Organization (WHO) declared the Coronavirus Disease 2019 (COVID-19) pandemic on March 11, 2020 and alerted all governments to prepare emergency plans for COVID-19. After this important notice, almost all health societies published their recommendations to guide patients and clinicians in how to behave during the pandemic. On March 17, 2020 the American Society for Reproductive Medicine (ASRM) recommended that the all assisted reproductive technology (ART) treatments and non-urgent diagnostic procedures should be postponed except for some cases which are currently "in-cycle" or who require urgent stimulation and cryopreservation, because of the unknown effect of COVID-19 on fertility, pregnancy, and fetus [1]. The European Society of Human Reproduction and Embryology (ESHRE) suggested similar recommendations to the ASRM on March 19, 2020 [2]. In Turkey, the first case was detected and declared on the March 10, 2020. Due to these societies' recommendations and being a part of the pandemic, many fertility centers in Turkey did not start any type of new ART treatment.

The updated version of the recommendations for ART has been published since March 2020. The latest version of ESHRE's guidelines recommended to restart ART under some rules and conditions [2]. Although the advice to return to normal daily practice has been offered [3], the risk of infection during the delivery of reproductive care; the possibility of COVID-19 contamination, and the effect of COVID-19 on the fetus and neonate are still unclear.

Many predisposing factors have been described for COVID-19. According to knowledge, there are no reports about whether or not ART is a predisposing or prognostic factor for COVID-19. In this report, we investigate 
the association between ART and COVID-19.

\section{Material-Methods}

After the ethical committee approval and the permission from the Republic of Turkey Ministry of Health, the data of 17 infertility patients who treated with ART between 01 and 11 March 2020 at a Reproductive Endocrinology and Infertility unit of a tertiary center were collected retrospectively. These patients were contacted and asked if they wanted to participate in the study and if they were positive for COVID-19 or had a symptom of the illness. All of the patients agreed to participate and answered the questions.

The SPSS 21.0 program was used in the statistical analysis of the data. The variables were expressed as mean \pm standard deviation $(\mathrm{X} \pm \mathrm{SD})$, and the percent (\%) value was calculated for variables determined by count. The "p" significance value was taken as 0.05 in all the statistical analyses.

\section{Results}

In the present study, 17 infertile women who were treated with ART at the fertility center between 1 and 11 March 2020 were contacted and all agreed to be included to the study. All of the treatments for the patients were initiated before the declaration of the Covid-19 outbreak. New cycles had not been initiated due to the recommendations of the international and national societies. The mean age of the patients was $34.35 \pm 4.24$ (minimum 26; maximum 41) years old and the duration of infertility was $6.41 \pm 5.27$ (minimum 1; maximum 18) years. The mean time of interview after the end of ART was $46.7 \pm 18.3$ days. As seen in Table 1; ovarian stimulation, oocyte pick-up, and intracytoplasmic sperm injection (ICSI) were used for all patients. Embryo transfer was performed on 9 (52.9\%) patients and clinical pregnancy was detected in 4 of the 9 patients after the ART procedure. At the time of the interview, none of the infertile women had any symptoms of COVID-19, such as fever, cough, headache, myalgia, fatigue, sore throat, diarrhea, acute loss of smell or taste, or a confirmed diagnosis from a positive test result for COVID-19 nucleic acids by realtime fluorescence reverse transcription-polymerase chain reaction (RT-PCR). No statistical analysis methods could be performed because a suspected or diagnosed COVID-19 case was not detected in the study group.

\section{Discussion}

Many predisposing and prognostic factors for COVID-19 have been identified. Higher age [4], male gender, hypertension, diabetes, cardiovascular disease, chronic lung disease, cancer [5-8], acute cardiac injury, and acute kidney injury [9] have all been described as effective factors on COVID-19. Despite the increasing knowledge on COVID-19, the uncertainty around special illness or treatments, like immunosuppressors or modulator agents, still continues. There has been conjecture whether or not Inflammatory Bowel Disease (IBD), which is a type of chronic immune-inflammatory disease, has any effect on, or relation with, COVID19. There have been no reports to suggest that Covid-19 occurs more frequently in IBD patients than in the general population [10], and while there are no current data on immune modulators for psoriasis [11] or the prolonged immunosuppressor treatments for organ transplant recipients [12], these conditions could cause a higher risk for this viral illness.

Gonadotropin-releasing hormone antagonist or agonists, clomiphene citrate, gonadotropins, progesterone, and human chorionic gonadotropin are the type of agents that are used in ART modalities. While these agents have been used for making a controlled ovarian hyperstimulation, promoting the development of the follicles, improving embryo implantation and pregnancy, they could cause supraphysiologic hormone levels that may be related with new clinical effects on a woman body. The high hormone levels during ART could cause some rare side effects in a long term period. It has been concluded that a women who applied to ART at any time should be closely monitored for the possibility of the complications of ART.

All new fertility treatments, except some cases, were postponed due to the key recommendations of the fertility societies since the middle of March 2020 [1,2]. After the prolonged lockdown of ART, it has been advised that fertility treatments for infertile couples can restart [3]. To provide fertility care in safe conditions, new recommendations have also been published [13]. According to our knowledge, only one patient pregnant after ART has been reported as testing positive for COVID-19 [14]. Although it was reported that 7 pregnant 
women died due to severe COVID-19 disease and one of these cases achieved pregnancy with ART due to the age-related infertility [14], another report showed that pregnant women are not at higher risk of COVID-19 compared with the general population [15]. It is still unknown whether or not ART could be a potential risk or prognostic factor for COVID-19. It has been shown that women are less likely to be positive for COVID 19 and that their mortality rate is less than men's $[16,17]$. It has also been hypothesized that this statistically significant difference has been caused by the role of the protective effect of estrogen (18). Furthermore, the use of conjugated equine estrogens [18] has been proposed to regulate the immune response to COVID19 by activating the estrogen receptors. These receptors are known as transcription factors regulating the development of immune cells and the pathways of the immune system [19]. Despite the recommendation concerning the use of estrogen for the treatment of COVID-19, the beneficial or harmful estrogen level is undetermined. The estrogen and progesterone levels in a stimulated cycle could be 10 times greater than the hormone levels in natural menstrual cycles [20]. It is doubtful that such a high level of hormones may still have an immunomodulatory role. To evaluate this relationship, the patients, who had been treated with ART recently, could provide a great opportunity to gain insight about the association between ART and COVID-19. In our study, the 17 infertile couples who applied for infertility treatment between March 1, 2020 and March 10, 2020 were evaluated, contacted and asked if they were positive for COVID-19 or had any symptoms of the virus. While we were talking to all cases, none of them were suspected as having, or diagnosed with, COVID-19. This result cannot show whether ART treatment may be a predisposing or prognostic factor for COVID-19. In addition, the patients who achieved pregnancy by ART treatment did not have any medical problem related with COVID-19. Almost all patients were below 40 years old, and it is well known that COVID-19 especially affects people who are older. It may be speculated that the women did not show any significant signs of COVID-19 due to the young ages in our study. All pregnancies were almost in the first trimester, so it could not be said whether pregnant women may or may not be at a higher risk of COVID 19 at different stages of the pregnancy period.

The main limitations of this study were that these results are only from a single fertility center, the number of participants is very low and there were no COVID-19 positive case to compare any findings between COVID-19 positive and negative patients. The other limitation was that at the time when we reached the patient, she may have been in the incubation period of COVID-19, so the case of a positive COVID-19 diagnosis would have been after the interview.

In conclusion, we want to bring attention to the potential risk of COVID-19 infection in patients who have been treated with ART. With the return to normal practice life, all fertility societies' guidelines should be followed carefully, all patients should be evaluated for COVID-19 during pre, per and post-treatment time for continuing ART treatments and protecting the patients from the side effects of ART, especially with the possible, but undefined, predisposition factor to COVID-19

\section{Author Contribution:}

YT contributed to data collection and analysis, manuscript writing, and revision. YK contributed to study design, manuscript writing, and revision. BT contributed to literature review, manuscript writing, and revision. TT contributed to data collection and analysis. CK contributed to study design, data analysis, and literature review.

CONFLICTS OF INTEREST: The authors have no conflicts of interest.

\section{References:}

1. American Society for Reproductive Medicine (ASRM). Patient Management and Clinical Recommendations During the Coronavirus (COVID-19) Pandemic. https://www.asrm.org/globalassets/asrm/asrmcontent/news-and-publications/covid-19/covidtaskforce.pdf. Accessed 25.05.2020.

2. ESHRE Guidance on Recommencing ART Treatments. https://www.eshre.eu/Home/COVID19WG. Accessed 25.05.2020. 
3. Updated Treatment Policy in Light of COVID-19 Updated April 29, 2020. https://www.embryo.net/covid19. Accessed 25.05.2020.

4. Liu W, et al. Analysis of factors associated with disease outcomes in hospitalized patients with 2019 novel coronavirus disease. Chin Med J 2020;133:00-00. doi: 10.1097/CM9.0000000000000775

5. Jordan RE, et al. Covid-19: risk factors for severe disease and death. BMJ. 2020;368:m1198. Published 2020 Mar 26. doi:10.1136/bmj.m1198

6. Wu Z, et al. Characteristics of and important lessons from the coronavirus disease 2019 (COVID-19) outbreak in China: Summary of a Report of 72314 Cases From the Chinese Center for Disease Control and Prevention [published online ahead of print, 2020 Feb 24]. JAMA. 2020;10.1001/jama.2020.2648. doi:10.1001/jama.2020.2648

7. Huang R, et al. Clinical findings of patients with coronavirus disease 2019 in Jiangsu province, China: A retrospective, multi-center study. PLoS Negl Trop Dis. 2020;14(5):e0008280. Published 2020 May 8. doi:10.1371/journal.pntd.0008280

8. Wang D, et al. Clinical characteristics of 138 hospitalized patients with 2019 novel coronavirus-infected pneumonia in Wuhan, China [published online ahead of print, 2020 Feb 7]. JAMA. 2020;323(11):1061-1069. doi:10.1001/jama.2020.1585

9. Wang X, et al. Comorbid chronic diseases and acute organ injuries are strongly correlated with disease severity and mortality among COVID-19 patients: A Systemic Review and Meta-Analysis. Research (Wash D C). 2020;2020:2402961. Published 2020 Apr 19. doi:10.34133/2020/2402961

10. Monteleone G, et al. Are patients with inflammatory bowel disease at increased risk for Covid-19 infection? [published online ahead of print, 2020 Mar 26]. J Crohns Colitis. 2020;jjaa061. doi:10.1093/eccojcc/jjaa061

11. Lebwohl M, et al. Should biologics for psoriasis be interrupted in the era of COVID-19?. J Am Acad Dermatol. 2020;82(5):1217-1218. doi:10.1016/j.jaad.2020.03.031

12. Hsu JJ, et al. COVID-19 in a high-risk dual heart and kidney transplant recipient [published online ahead of print, 2020 Apr 21]. Am J Transplant. 2020;10.1111/ajt.15936. doi:10.1111/ajt.15936

13. Alviggi C, et al. COVID-19 and assisted reproductive technology services: repercussions for patients and proposal for individualized clinical management. Reprod Biol Endocrinol. 2020;18(1):45. Published 2020 May 13. doi:10.1186/s12958-020-00605-Z

14. Hantoushzadeh S, et al. Maternal death due to COVID-19 [published online ahead of print, 2020 Apr 28]. Am J Obstet Gynecol. 2020;S0002-9378(20)30516-0. doi:10.1016/j.ajog.2020.04.030

15. https://www.who.int/news-room/q-a-detai l/q-a-on-covid -19-pregnancy-child birth -and-breast feeding. Accessed 25.05.2020..

16. Chen N, et al. Epidemiological and clinical characteristics of 99 cases of 2019 novel coronavirus pneumonia in Wuhan, China: a descriptive study. Lancet. 2020;395(10223):507-513. doi:10.1016/S01406736(20)30211-7

17. China CDC Weekly: Vital Surveillances: The epidemiological characteristics of an outbreak of 2019 novel coronavirus diseases (COVID-19) - China, 2020. The Novel Coronavirus Pneumonia Emergency Response Epidemiology. 2020; 2(8): 113-122.

18. Suba Z. Prevention and therapy of COVID-19 via exogenous estrogen treatment for both male and female patients. J Pharm Pharm Sci. 2020;23(1):75-85. doi:10.18433/jpps31069

19. Kovats S. Estrogen receptors regulate innate immune cells and signaling pathways. Cell Immunol. 2015;294(2):63-69. doi:10.1016/j.cellimm.2015.01.018 
20. Joo BS, et al. Serum estradiol levels during controlled ovarian hyperstimulation influence the pregnancy outcome of in vitro fertilization in a concentration-dependent manner. Fertil Steril. 2010;93(2): 442-6.

Table 1: Clinical Characteristics of the Participants

\begin{tabular}{llll}
\hline & & N & \% \\
\hline OPU & & 17 & 100.0 \\
ET & Performed & 9 & 52.9 \\
& Cancelled & 8 & 47.1 \\
Clinical Pregnancy Rate / Embryo transfer & & 4 & 44.4 \\
Known as positive for COVID-19 after ART & Yes & 0 & 0.0 \\
& No & 17 & 100.0 \\
Any symptom of COVID-19 after ART & Yes & 0 & 0.0 \\
& No & 17 & 100.0 \\
\hline
\end{tabular}

ART: Assisted Reproductive Treatment; COVID-19: Coronavirus Disease 2019; ET: Embryo Transfer; OPU: Oocyte Pick-up; ICSI: Intracytoplasmic Sperm Injection

\section{Hosted file}

table.docx available at https://authorea.com/users/348108/articles/478889-the-relationshipof-assisted-reproductive-technology-with-covid-19-infection-in-women 